\title{
El impacto del terrorismo en el discurso político en España: un análisis de contenido de los discursos de los presidentes de Gobierno en los debates parlamentarios
}

\author{
The impact of terrorism on political discourse in Spain: A content analysis \\ of the Spanish Presidents' speeches at parliamentary debates
}

\author{
RAFAEL LEONISIO \\ Universidad del País Vasco (UPV-EHU)
}

\section{Cómo citar/Citation}

Leonisio, R. (2019). El impacto del terrorismo en el discurso político en España: un análisis de contenido de los discursos de los presidentes de Gobierno en los debates parlamentarios. Revista Española de Ciencia Política, 49, 129-149.

Doi: https://doi.org/10.21308/recp.49.06

\section{Resumen}

Esta nota de investigación trata de analizar los determinantes de la relevancia del terrorismo en el discurso político de las élites, mediante un análisis de contenido de las intervenciones parlamentarias de los presidentes de Gobierno español en las sesiones de investidura y debates sobre el estado de la nación. De esa manera se muestra el peso cuantitativo que el terrorismo ha tenido en los discursos de los jefes de Gobierno en España. Además de describir el peso de esta cuestión, se apuntan una serie de hipótesis respecto a los cambios en la relevancia en dichos discursos, y se contrastan provisionalmente. En primer lugar, el texto apunta a que las treguas de los grupos terroristas tienen cierta influencia, incrementando la importancia que las élites políticas otorgan a este fenómeno. Segundo, los atentados especialmente relevantes también hacen crecer su presencia en los discursos políticos. En tercer lugar, la influencia es inversa en lo que respecta a los acuerdos formales para excluir al terrorismo de la competición política (por ejemplo, el Pacto de Ajuria-Enea), ya que reduce la relevancia de esta cuestión. Finalmente, la opinión pública también cuenta, de modo que cuanto más gente considera el terrorismo como un problema, más hablan los presidentes españoles del mismo. La nota trata de cubrir un hueco en la investigación, ya que el análisis cuantitativo del discurso de las élites políticas respecto al terrorismo ha sido en general pasado por alto. Metodológicamente, combina análisis de contenido de textos políticos, datos de encuesta y datos contextuales respecto a los atentados terroristas y treguas de ETA.

Palabras clave: terrorismo, discurso político, análisis de contenido, España, ETA. 


\begin{abstract}
This research note aims to analyze the determinants of terrorism saliency in elites' political discourse by means of a content analysis of the Spanish Presidents' speeches at the investiture sessions and the debates on the general political situation of Spain in the Spanish Parliament. Thus, it is shown the quantitative weight terrorism has had in these speeches. Apart from describing the weight of this issue, the article points out a series of hypotheses regarding changes of the saliency in these speeches and it also tries to check them provisionally. The text first states that ceasefires declared by terrorist gangs have some influence, raising the importance politicians give to this phenomenon. Second, especially relevant attacks also make terrorism grow its presence in political speeches. Third, formal agreements to exclude terrorism from party competition (such as the Ajuria-Enea Pact) have the opposite influence, for they reduce the saliency of this issue. Finally, public opinion matters: The more people consider terrorism as a problem, the more Spanish Presidents talk about it. The note tries to fill a gap in research, because the quantitative analysis of political elites' discourse about terrorism has been largely neglected. Methodologically, it combines content analysis of political texts, survey data and contextual data regarding ETA's terrorist attacks and ceasefires.
\end{abstract}

Keywords: terrorism, political discourse, content analysis, Spain, ETA.

\title{
INTRODUCCIÓN
}

El terrorismo o la violencia política, en general, son en la actualidad un fructífero campo de investigación en ciencias sociales, algo en consonancia con la importancia que, tras los ataques del 11 de septiembre de 2001, ha adquirido este fenómeno en la sociedad global ${ }^{1}$. Son muchas y variadas las cuestiones estudiadas en relación con este tema, y el discurso de los diferentes actores políticos es una de ellas, aunque es cierto que no una de las más comunes. Así, no son pocos los académicos que han estudiado el discurso de los propios terroristas o sus organizaciones afines (Alonso, 2003, 2016; Mcevoy et al., 2004; McGovern, 2004; Reinares, 2001), el discurso de las élites o de los actores políticos en general en relación al terrorismo y la violencia política (Herschinger, 2013; Geifman, 2013; Thorup, 2009), o de ambos a la vez (Martín-Peña y Opotow, 2011; Whiting, 2012). Sin embargo, estas aproximaciones se han hecho en su mayoría desde un punto de vista cualitativo, por lo que en general no existen apenas aproximaciones cuantitativas a este fenómeno.

Este trabajo trata de cubrir parte de ese vacío en la investigación con un estudio cuantitativo del discurso político en España que ha tenido como objeto el terrorismo

1. Si bien existe en general consenso académico sobre lo que es la violencia política no es así sobre qué significa el terrorismo. Este texto no entrará en discusión, para la cual se pueden consultar Herschinger (2013); Schmid (2010), o Weinberg et al. (2011). Más concretamente, para una definición de terrorismo en contraste con la violencia ejercida por las guerrillas véase De la Calle y Sánchez-Cuenca (2011). 
y los diversos aspectos derivados de él (atentados, presos, víctimas, políticas antiterroristas, negociaciones, etc.). La España democrática ha sido golpeada tanto por el terrorismo de extrema izquierda (GRAPO) y el yihadista como, sobre todo, por el ejercido por ETA y sus organizaciones afines, que ha sido el más letal con casi novecientas personas asesinadas y un centenar de secuestrados. Además, ETA ha provocado más de 20000 víctimas directas en sus atentados (heridos y damnificados) en toda España (Llera, 2013: 8), cuantiosos daños materiales y varios miles de personas amenazadas, que durante un periodo de su vida estuvieron obligadas a vivir con escolta policial, lo que Gesto por la Paz definió como «violencia de persecución». Respecto a este último tipo de violencia, hay que decir que según López Romo (2015: 104-106), hasta 2001 habría habido unos 15649 amenazados. Para Gesto por la Paz (Llera 2013: 8) habrían sido unos 40000 en todo el periodo.

ETA, organización autodisuelta en 2018, fue el segundo grupo terrorista más letal de Europa Occidental, tras el IRA Provisional (Sánchez-Cuenca, 2010). Creada en 1959, combatió violentamente al franquismo desde sus inicios a pesar de que su primera víctima mortal data de 1968 (Domínguez y Fernández, 2018). Durante la dictadura y hasta la celebración de las primeras elecciones democráticas en junio de 1977 su número de asesinatos fue muy bajo (66 personas, un 7,8 \%) en comparación con lo que vendría después ${ }^{2}$. Observando el gráfico 1 podemos obtener, entre otras, dos conclusiones. En primer lugar que ETA ha sido, con mucha diferencia, la organización terrorista más letal que ha actuado en España. De los alrededor de 1300 muertos por terrorismo en España, aproximadamente dos tercios han sido causados por ETA y sus organizaciones afines. Además, excluyendo los atentados islamistas del 11-M y de las Ramblas el porcentaje subiría hasta casi un $80 \%$. En segundo lugar, el gráfico muestra con claridad cómo a partir de la restauración de la democracia el terrorismo se dispara. Si bien en los años 1974, 1975, 1976 y 1977 (con alrededor de 20-25 muertos por año, la mayoría cometidos por ETA) el terrorismo golpea con más fuerza que en los años anteriores (solo hay seis muertes hasta 1972 y 8 en 1973), es precisamente a partir de 1978, con la legislatura constituyente a pleno rendimiento, cuando comienza una gran escalada que durará tres años con 86 muertos (65 de ETA) en 1978, 111 (78) en 1979 y 127 (96) en 1980. Y aunque a partir de los primeros ańos ochenta se reduce el número de asesinatos, no se recuperan hasta inicios del siglo XXI los números más bajos de los primeros años setenta (salvo excepciones). Así, de 1968 a 1975 ETA asesinó a 46 personas (5,75 por año), mientras que de 1976 hasta su final en 2011 segó la vida de 804 (22,3 de media anual $)^{3}$.

2. Datos obtenidos de The victims of ETA dataset del Instituto Juan March.

3. Si tomásemos como punto de corte 1977 , serían 73 (7,3 al año) para la dictadura franquista y $777(22,9)$ para el periodo democrático. En cualquier caso hay que tener en cuenta que a partir de 2003, y debido a la eficacia de la acción policial, ETA es una organización con muy poca capacidad de atentar. De hecho, su último asesinato antes de la bomba de la T4 con la que rompió la tregua de 2006 es de mayo de 2003. Aún así, desde esa fecha hasta su final solo es capaz de asesinar a nueve personas, es decir, baremos similares a los del tardofranquismo. 
GRÁfICO I.

Muertes POR TERrorismo EN EsPaña (I968-20I7)

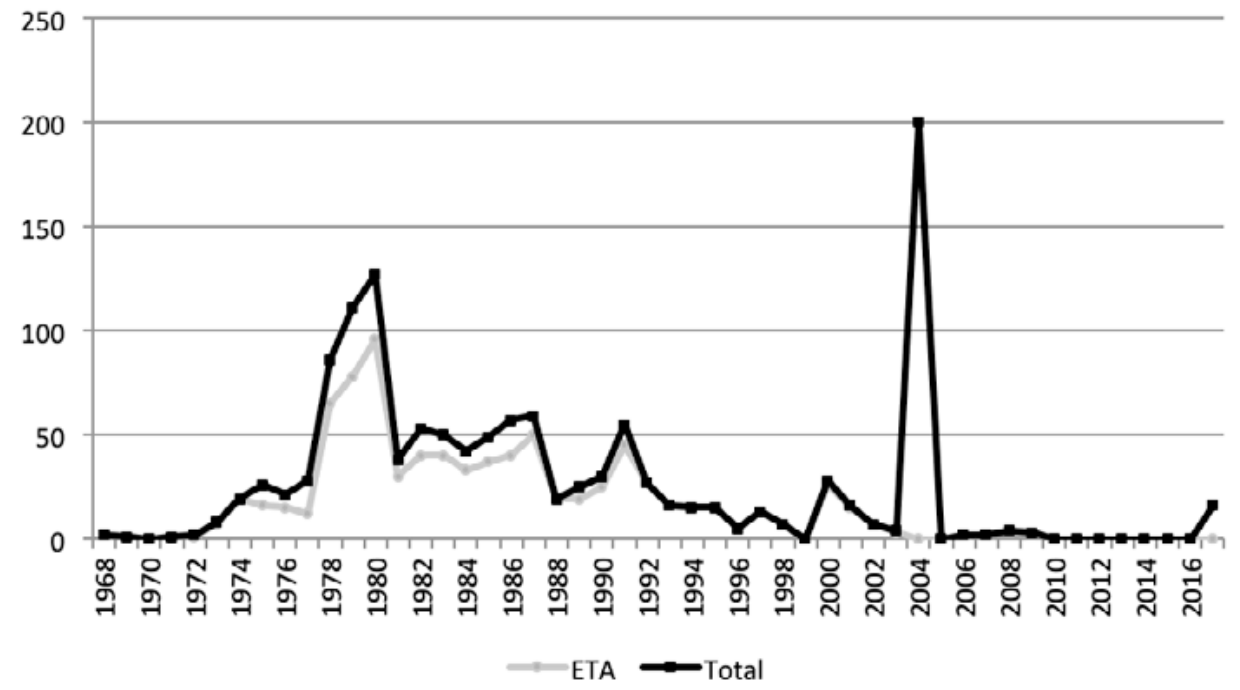

Fuente: elaboración propia a partir de los datos del Ministerio del Interior.

Por tanto, ETA ha sido básicamente una organización que se ha enfrentado a la democracia española, contraponiendo a esta su proyecto de un País Vasco independiente y socialista compuesto por las actuales comunidades autónomas españolas de Euskadi y Navarra y los territorios vascos del suroeste de Francia (englobados en el departamento de Pirineos Atlánticos, en la región de Aquitania) ${ }^{4}$. Esta anómala situación supuso un grave problema para la consolidación de la democracia tanto en el País Vasco como en el resto de España, ya que tuvo como consecuencia, entre otras muchas, la generalización del miedo a participar en política y a expresar opiniones, sobre todo en Euskadi (Domínguez, 2003; Llera y Leonisio 2017), y la distorsión de la representación democrática debido al asesinato de representantes políticos. Así, durante la transición fueron asesinados en el País Vasco varios miembros de UCD, partido en el Gobierno de España en aquel momento. Además, a partir del año 1995 representantes políticos y militantes de PP, PSOE, UPN y UA fueron acosados, dañadas sus propiedades y en última instancia, alrededor de tres decenas fueron asesinados (algunos de ellos en el resto de España). En general, fueron concejales de pequeños pueblos, pero ETA también asesinó a conocidos líderes políticos como el exvicelehendakari socialista Fernando Buesa o el parlamentario vasco Gregorio Ordóńez.

4. Para bibliografía sobre la historia de ETA y, en general, su entorno, véase Garmendia (1980); Jáuregui (1981); Sulivan (1988); Domínguez (1998); Elorza (2000); Mees (2003); Muro (2008); Watson (2008), y Casquete (2009). Para contribuciones más recientes, véase Domínguez (2012); Fernández Soldevilla y López Romo (2012); Fernández Soldevilla (2013, 2016); Whitfield (2014), y Leonisio et al. (2017). 
La distorsión que el fenómeno terrorista ha supuesto tanto para la sociedad vasca como para la española ha tenido su correlato en la preocupación que ha suscitado en ambas opiniones públicas ${ }^{5}$. Como puede verse en el gráfico 2, con diferentes altos y bajos dependiendo del contexto (crisis económicas o treguas de la propia ETA), los datos demoscópicos nos muestran que el terrorismo ha sido una cuestión siempre presente en las preocupaciones de los españoles, en general, y de los vascos, en particular, con un importante pico coincidiendo con el cambio de siglo. A partir de ese momento, el descenso es continuado en ambas opiniones públicas, y se desploma hasta niveles insignificantes con la crisis económica y el abandono de las armas por parte de ETA. En 2013, por ejemplo, tan solo el 0,5\% de los españoles y el $2 \%$ de los vascos decían que el terrorismo era uno de los tres problemas que más les preocupaban. Sin embargo, como se aprecia en el gráfico, hasta fechas recientes ha sido una cuestión muy presente en ambas opiniones públicas, reflejo de la distorsión que la violencia terrorista ha provocado en la democracia española. Puede llamar la atención el nulo efecto de los atentados islamistas de Barcelona y Cambrils de agosto de 2017. Esto es efecto de usar siempre el último dato anual (diciembre), ya que la preocupación subió hasta un $15 \%$ en septiembre, para después volver a sus niveles habituales. El dato de 2015 es también excepcional. El mes anterior había sido un $0,8 \%$ y el mes siguiente bajó hasta un $5,8 \%$, y en todo 2016 se situó en el 1-2\%. Ese dato excepcional se debe, sin duda, a los atentados de París de noviembre de 2015, en los que murieron unas 130 personas.

Así pues, hasta la segunda mitad de la década de los años 2000, el terrorismo fue una de las principales preocupaciones tanto de los españoles como de los vascos. Este dato es de sobra conocido, ya que la opinión pública respecto al terrorismo en España ha recibido bastante atención por parte de la investigación (Criado, 2017; Llera, 1992, 2012, 2013). Sin embargo, como hemos dicho un poco más arriba, el discurso de las élites, desde un punto de vista cuantitativo, no ha sido estudiado de manera satisfactoria, tanto en España como en general en el resto de países que han sufrido violencia política. Así, en este punto, la pregunta de investigación que nos hacemos es: ¿cuál ha sido la atención que a lo largo de todo el periodo democrático han tenido las élites políticas españolas hacia al terrorismo? Como se verá más detalladamente en el siguiente apartado, para obtener un indicador que mida la relevancia del terrorismo en el discurso político hemos realizado un análisis de contenido de los discursos de los candidatos a presidentes de Gobierno en los debates de investidura (excepto Pedro Sánchez en 2016, todos fueron elegidos) y de los presidentes en los debates sobre el estado de la nación en el Congreso de los Diputados, y se ha obtenido el porcentaje de tiempo de los mismos dedicado a todas las cuestiones relacionadas con el problema del terrorismo.

5. Tanto el CIS como el Euskobarómetro preguntan por las 3 principales preocupaciones de los ciudadanos. El dato que utilizamos en nuestros cálculos es la suma de la multirrespuesta, es decir, el porcentaje de espańoles o vascos que dice estar preocupado por el terrorismo en primer, segundo o tercer lugar. El Euskobarómetro pregunta explícitamente por la preocupación, mientras que el CIS lo hace por los tres principales problemas que hay en España. En aras de la brevedad hablaremos en todo el texto de preocupaciones cuando nos refiramos a esta cuestión. 
GRÁfICO 2.

EVOLUCIÓN DE LA PREOCUPACIÓN POR EL TERRORISMO EN LAS OPINIONES PÚBLICAS VASCA Y ESPAÑOLA

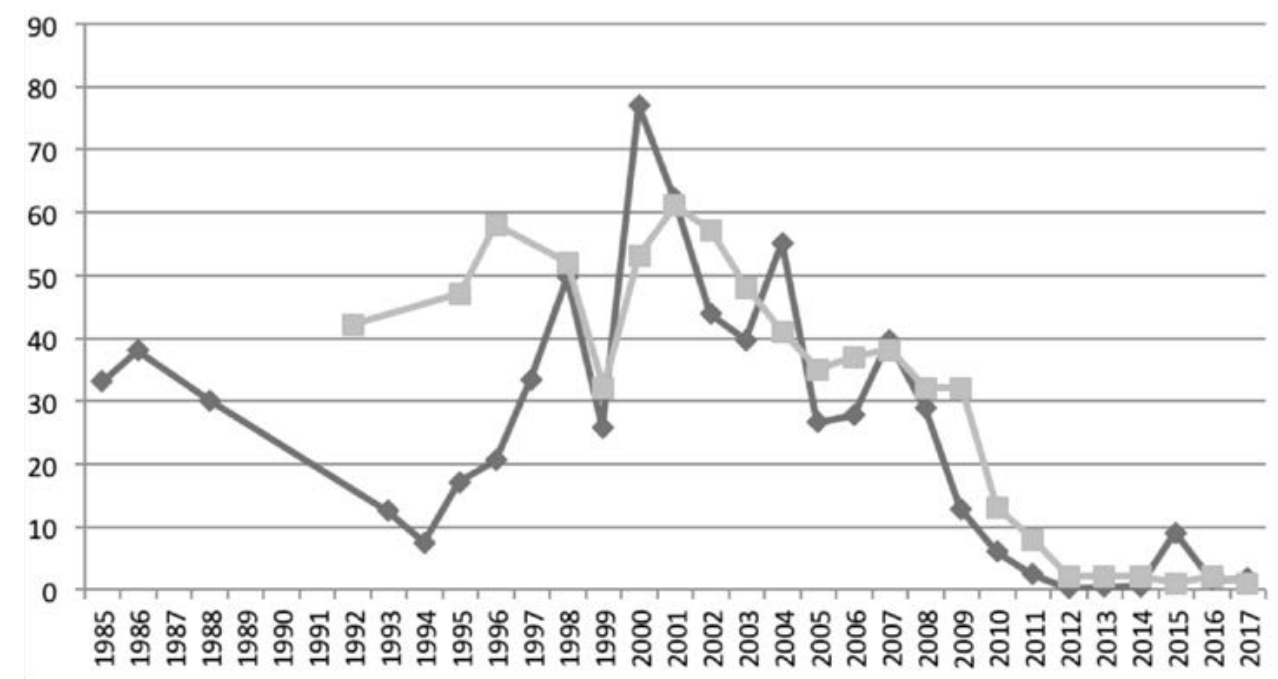

— España (CIS) —- Euskadi (Euskobarómetro)

Fuente: elaboración propia a partir de los datos del CIS y del Euskobarómetro.

Nota: siempre se toma el último dato anual disponible de cada serie.

De esta manera, sabiendo el porcentaje destinado a esta cuestión, hemos obtenido un proxy tanto de la importancia que la violencia ha tenido para las élites políticas españolas como del tiempo que le han dedicado en su práctica política desde el inicio del periodo democrático hasta nuestros días. Aun siendo este dato ya de por sí interesante, esta nota de investigación pretende ir más allá de la cuantificación descriptiva y busca una explicación a la variación de dicho discurso a lo largo del tiempo. Debido al escaso número de datos de los que disponemos en este momento, el análisis será tentativo y servirá sobre todo para apuntar algunas hipótesis que deberán ser contrastadas en un futuro, cuando haya más material disponible después de desarrollar plenamente esta línea de investigación. Finalmente, la última aportación de este trabajo será proveer en el anexo los datos brutos de la variable dependiente, para que así puedan estar a disposición de cualquier investigador que quiera utilizarla tanto como variable dependiente, para replicar o mejorar el análisis que aquí se hace, como variable independiente en otro tipo de análisis. Tras esta introducción, el texto se estructura en tres apartados. El siguiente explica someramente la metodología que se ha utilizado para codificar los discursos de los presidentes de Gobierno. En el tercero se presentan los resultados, los cuales se analizan comprobando de manera provisional cuatro diferentes hipótesis. Finalmente, en el cuarto y último punto se apuntan una serie de conclusiones. 


\section{METODOLOGÍA: EL ANÁLISIS DE CONTENIDO}

Una forma de responder a nuestra pregunta de investigación es realizar un análisis de contenido de dichos discursos para, de esa manera, obtener el porcentaje de tiempo dedicado en estos a diversos aspectos relacionados con la violencia terrorista. Esta forma de medir el objeto de estudio permite crear una variable cuantitativa que puede visualizarse y, además, ser tratada estadísticamente.

Cuando se utiliza la metodología del análisis de contenido, una de las decisiones más importantes es elegir los textos que se van a analizar. Para estudiar el discurso político en España es ingente la documentación de la que a priori se podría hacer uso: discursos de líderes en mítines u otros contextos, intervenciones parlamentarias, resoluciones congresuales o programas electorales. Ante la imposibilidad de acceder a todo el universo de datos, se hace necesaria la elaboración de una muestra. En este caso, al ser este un texto exploratorio de la potencialidad que este tipo de análisis puede tener, hemos elegido los discursos parlamentarios de los presidentes de Gobierno en dos tipos de debates: investidura y sobre el estado de la nación. Hemos escogido a los presidentes por ser los líderes con más capacidad para marcar la agenda y, por tanto, ser sus discursos la mejor aproximación que podemos obtener de la importancia que la élite política española ha dado al terrorismo en cada periodo. Teniendo en cuenta que nuestro objetivo es conocer el porcentaje de tiempo dedicado al terrorismo, es condición indispensable que los textos sean generalistas, es decir, que no exista a priori ningún tema sobre el que el orador esté obligado a hablar y que por tanto dedique tiempo a diferentes cuestiones según su elección. De esta manera podemos medir la importancia que se otorga una determinada cuestión. Es esta una condición que cumplen los dos tipos de texto que vamos a analizar.

Desde 1977 ha habido en España quince sesiones de investidura ${ }^{6}$ y veinticinco debates sobre el estado de la nación, los cuales se han celebrado anualmente desde 1983, excepto cuando ha habido recientemente una sesión de investidura. Desde 1981, siempre se ha celebrado una sesión de tipo generalista todos los ańos (excepto en 1990, 2017 y 2018), lo que nos permite abarcar prácticamente año por año el discurso de los diferentes presidentes de Gobierno y, por tanto, ser muy exhaustivos en lo que se refiere a la evolución temporal ${ }^{7}$.

6. Suárez (1977 y 1979), Calvo Sotelo (1981), González (1982, 1986, 1989, 1993), Aznar (1996 y 2000), Rodríguez Zapatero (2004 y 2008), Rajoy (2011 y dos en 2016) y Sánchez (2016). La primera de Suárez no está disponible en formato legible por ordenador, por lo que queda fuera del análisis. También lo hace la primera de Rajoy en 2016 por estar su discurso ya bien representado con la segunda.

7. Hemos decidido analizar solo la primera intervención porque estimamos que representa mejor la posición del presidente de turno, al estar las réplicas y contrarréplicas más "contaminadas» por el propio contexto del debate. Estimamos, por tanto, que la primera intervención es la que más se aproxima al discurso «oficial» del presidente en cada momento. Los discursos parlamentarios han sido obtenidos en la página web del Congreso de los Diputados: http://www.congreso.es. 
Los textos se han codificado manualmente con la ayuda del programa de análisis de contenido NVivo. La codificación manual consiste en leer los textos y señalar al programa qué partes de los mismos se corresponden a los temas elegidos (en nuestro caso solo uno, el terrorismo). Una vez codificados, el programa calcula el porcentaje de texto dedicado a esas cuestiones por medio del recuento de frecuencias de las unidades de registro codificadas ${ }^{8}$. El análisis de contenido manual es la metodología más utilizada para estudiar los textos políticos. Sus desventajas, frente a otras propuestas desde un punto de vista más o menos computerizado y automatizado, serían su gran coste, tanto de tiempo como de dinero, a la hora de abordar una gran cantidad de textos y su falta de fiabilidad'. Por el contrario es la técnica que mejor asegura la validez de los resultados, además de dar a estos mayor precisión $^{10}$.

\section{ANÁLISIS}

Lo primero que llama la atención del gráfico 3 son las constantes subidas y bajadas del peso del terrorismo en el discurso de los presidentes de Gobierno españoles. Así, por ejemplo, si en 1994 Felipe González dedica tan solo un 0,4 \% de su tiempo al terrorismo en el debate sobre el estado de la nación de ese año, en 1995 el porcentaje sube hasta un 17,1 \%. En el caso de Aznar, Zapatero y Rajoy se repite el mismo patrón, por lo que parece que el terrorismo no era un tema con una importancia "fija» en sus discursos, sino que dependiendo de las circunstancias adquiría más o menos notoriedad. La tabla $1^{11}$ nos proporciona información de la media de tiempo que cada presidente dedicó al terrorismo en sus intervenciones parlamentarias en las sesiones de investidura y del debate del Estado de la nación. Lo que más llama la atención es la gran diferencia entre la media de Rajoy $(0,9 \%)$ y los otros tres presidentes. La clave parece estar en el fin de ETA, que justo abandonó el terrorismo poco tiempo antes de que se celebrasen las elecciones generales que supusieron el inicio de la era Rajoy en el Gobierno. Así, parece que la más

8. En lo que respecta al programa NVivo, este cuenta los caracteres (espacios incluidos). En caso de analizar videos o audios lo que se codifica (y luego el programa recopila) son los segundos.

9. Para despejar las dudas sobre la posible falta de fiabilidad de nuestra codificación manual véanse la tabla A2 y el gráfico A1 en el anexo.

10. En análisis de contenido el término «validez» significa que la técnica está midiendo efectivamente lo que dice que mide, mientras que "fiabilidad" se refiere a que el procedimiento debe rendir los mismos resultados, aunque lo apliquen diferentes personas y en diferentes momentos. En cualquier caso, no es este el lugar para discutir las bondades o desventajas de las distintas técnicas de análisis de contenido. Para un contraste entre técnicas manuales y computerizadas véase Laver (2001); Volkens (2007), o Grimmer y Stewart (2013).

11. No mostramos los resultados para Suárez, Calvo Sotelo y Sánchez por tener de ellos solo un dato. 
global y difusa amenaza yihadista no merece tanta atención como la que tenía ETA para sus antecesores, de los cuales el que más tiempo le dedicó fue Aznar (casi un $10 \%)$, mientras que González y Zapatero lo hicieron de manera similar (6,2 \% y $6,6 \%$ respectivamente).

Dejando de lado el dato de Rajoy, especial por estar su mandato en diferentes circunstancias (es decir, sin ETA) que los presidentes anteriores, los resultados son coherentes con lo obtenido en investigaciones previas, donde se ha constatado que el terrorismo es más beneficioso, en términos electorales, para los partidos situados más a la derecha, y que por tanto estos tenderán a primar esta cuestión en su agenda política (Berrebi y Klor, 2008; Hetherington y Suhay, 2011; Kibris, 2011; Criado, 2017). Esto obviamente deberá ser comprobado con más datos para poder ser confirmado, por ejemplo comparando los discursos de los portavoces del PP frente a los de los partidos de izquierdas en el Parlamento, pero es algo que queda fuera del ámbito de esta nota. Por otro lado, es bien conocido que, como líder la oposición, Rajoy basó buena parte de su discurso en criticar la gestión del Gobierno de Rodríguez Zapatero en lo que respecta al terrorismo, lo que en parte iría en consonancia con que esta cuestión está más presente en partidos de derecha. De hecho, fue muy diferente la actitud de la oposición popular en el alto el fuego de ETA de 2006 y la del PSOE en la tregua de Lizarra de 1998-99. Mientras que en el primer caso la oposición fue muy beligerante con el Gobierno, situando el tema del terrorismo en un primer plano de la agenda pública, en el segundo el PSOE mantuvo una posición discreta sin poner en cuestión las decisiones gubernamentales.

\section{GrÁFICO 3.}

Porcentaje del discurso de los presidentes de gobierno españoles dedicado al TERRORISMO EN LAS SESIONES DE INVESTIDURA Y DEBATE SOBRE EL ESTADO DE LA NACIÓN EN EL Congreso de los Diputados (i979-20i6)

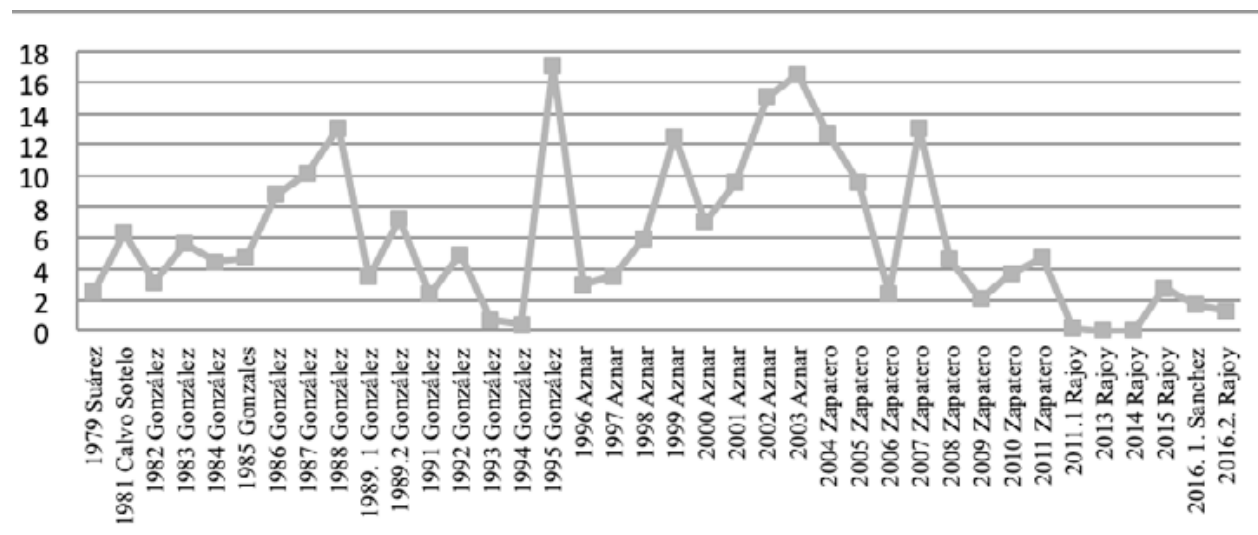

Fuente: elaboración propia. 
TABLA I.

Media de tiempo dedicado al terrorismo de CADA PRESIDENTE de GOBIERNO EN LOS DEBATES SOBRE EL ESTADO DE LA NACIÓN Y DE INVESTIDURA

\begin{tabular}{ll}
\hline González & 6,2 \\
\hline Aznar & 9,1 \\
\hline Zapatero & 6,6 \\
\hline Rajoy & 0,9 \\
\hline
\end{tabular}

Fuente: elaboración propia.

¿Qué hay detrás, además del fin de ETA y de la cuestión ideológica, tanto de esas medias como de las subidas y bajadas anuales que se ven en el gráfico 3? De entrada hay que descartar la hipótesis lógica de que, cuanto más golpee el terrorismo, más hablarán de él los políticos, en nuestro caso el presidente del Gobierno. La comparación entre González y Zapatero es bien elocuente, ya que si bien dedican, de media, un tiempo similar a hablar de esta cuestión, en el periodo de presidencia del primero hubo unos 460 muertos (una media de unos 35 por año) en acciones terroristas y diecinueve secuestros, mientras que durante la presidencia de Zapatero ETA (y ninguna otra organización) asesinó a 11 personas en Espańa (poco más de una persona de media anual) ${ }^{12}$. Lo mismo podemos decir de Aznar, quién dedicó más tiempo que Zapatero y también que González, aunque hubo más asesinatos en su época que en la del primero, pero no más que en la del segundo. Además, por ejemplo, Aznar dedicó más tiempo al terrorismo en los años 2002 y 2003 que en los dos ańos anteriores, en los cuales tuvo lugar la ofensiva etarra post-tregua de Lizarra (en el ańo 2000 hubo veintitrés muertos, la cifra más alta desde 1992, y en 2001 quince, no llegándose más a dichas cifras en los ańos posteriores en que ETA estuvo activa). ${ }^{13}$

Si en vez de personas asesinadas utilizamos el dato de acciones violentas, en general, la conclusión que obtenemos es la misma. He realizado una correlación entre los porcentajes de cada discurso dedicados al terrorismo y el número de acciones violentas provocadas por diversos grupos terroristas que se dieron en el año en el que este se pronunció $^{14}$. El resultado indica una ausencia total de relación $(\mathrm{R}=0,031$, sig. $=0,867, \mathrm{~N}=$ 31 ), es decir, que el hecho de que haya más o menos acciones violentas no hace crecer o disminuir el tiempo dedicado al terrorismo en los discursos de los presidentes.

Así pues, esto nos indica que las variables contextuales que explican la presencia del terrorismo en el discurso político no hay que buscarlas en una mayor o menor letalidad o intensidad del terrorismo (por lo menos en lo que respecta al caso espańol), sino en otras cuestiones diferentes. A continuación apuntaré cuatro hipótesis que

12. En la época de González la gran mayoría de los asesinados, 380 (82\%) fueron cometidos por ETA y sus organizaciones afines.

13. Datos obtenidos de The victims of ETA dataset y Llera (2013: 11).

14. Datos obtenidos de Llera (id.). 
trataré de contrastar de manera provisional con los escasos datos disponibles hasta el momento y que deberán ser aceptadas o rechazadas según se vaya avanzando en la investigación y obteniendo nuevas evidencias. Por tanto, este estudio es el primer paso hacia una investigación más completa que con el tiempo nos ayudará a obtener respuestas sobre las causas de la mayor o menor atención que los líderes políticos conceden al problema del terrorismo.

\section{Hipótesis 1. Treguas}

Una posibilidad es que cuando las organizaciones terroristas declaran una tregua los políticos fijan más su atención en el problema del terrorismo, ya que se abre una puerta para resolverlo. A lo largo de su historia, ETAm (única existente desde mediados de los ochenta) declaró cuatro treguas de cierta entidad: la tregua paralela a las conversaciones de Argel, durante los primeros meses de 1989; la tregua consecuencia del Pacto de Lizarra, entre septiembre de 1998 y noviembre de 1999; el alto el fuego declarado en marzo de 2006 y que finalizó en junio de 2007 (aunque es cierto que roto de facto en diciembre de 2006 con el atentado de la T4 en Barajas) y, finalmente, el alto del fuego de septiembre de 2010 que culminará en octubre de 2011 con el cese definitivo de su actividad violenta.

$¿$ Tienen como consecuencia las treguas declaradas por la banda terrorista un incremento en la atención de los presidentes de Gobierno hacia el terrorismo? La evidencia es mixta. El debate sobre el estado de la nación de 1989 se celebra en febrero, es decir, en medio de la tregua decretada por ETA y, como se puede comprobar en el gráfico, es el momento que menos se habla de terrorismo desde 1982. En cuanto a las sesiones de 2010 y 2011 también se producen en plena tregua y, aunque hay una subida en importancia desde el año anterior, esta no parece muy grande y, además, por ejemplo, desciende respecto a 2008. Sin embargo, las otras dos treguas sí parecen tener una cierta influencia. El dato de 1999, en plena tregua de Lizarra, es el más alto del presidente Aznar hasta ese momento y culmina una subida continuada desde 1996 (Aznar solo volverá a hablar más de terrorismo en 2002 y 2003), y si bien Rodríguez Zapatero responde en un principio al alto al fuego de ETA de 2006 con cierta indiferencia (en la sesión, tres meses después de la tregua, solo le dedica un 2,4\%), en la sesión de 2007, celebrada menos de un mes después de la ruptura, el dato se dispara hasta un $13 \%$, el máximo de esta etapa socialista.

¿Por qué unas treguas generan mayor atención y otras no? Quizá no es casualidad que en dos de ellas, las de $1998-99$ y 2006-07, se produjeron "procesos de paz» altamente debatidos y que ello haya hecho que la cuestión del terrorismo se dispare en el discurso de los presidentes. Recordemos que la tregua de Lizarra supuso una gran polémica porque fue paralela a la unidad de los partidos nacionalistas vascos, que se concretó en iniciativas como el pacto de legislatura entre el Gobierno Vasco (PNV-EA) y el brazo político de ETA (entonces EH) o la llamada Asamblea de Municipios de Euskal Herria o Udalbiltza, que fue la plasmación de la «institución única y soberana 
para el conjunto de Euskal Herria» que ETA reclamaba en su comunicado de tregua en septiembre de 1998 (Leonisio, 2015: 6-7). En este caso, los dos principales partidos españoles, PP y PSOE, estuvieron unidos contra dichas iniciativas. Sin embargo, no ocurrió lo mismo con la siguiente tregua, en la cual el PP se puso enfrente de los intentos de diálogo del Gobierno español con ETA, convocando incluso manifestaciones en contra de cualquier acercamiento. Por tanto, y a falta de una mayor evidencia, podemos concluir que cuando existen treguas y estas se asocian a diálogos o procesos de paz, la atención de los políticos sobre el terrorismo se incrementa de manera significativa, lo que se refleja en una mayor presencia de ese tema en sus discursos.

\section{Hipótesis 2. Atentados especialmente impactantes}

La presencia de ETA hizo del terrorismo un evento «cotidiano» para la ciudadanía española, en un proceso quizás semejante al que provoca en la actualidad el terrorismo yihadista en Europa, incluida España. Sin embargo, hubo algunos atentados que por sus especiales características (relevancia del asesinado, número y tipo de muertos, etc.) tuvieron un impacto especial, como el secuestro y asesinato de Miguel Ángel Blanco, por estar anunciado de antemano, o los atentados de Hipercor y el 11-M, por su carácter de masacre indiscriminada de civiles. Así pues, a pesar de que tanto ciudadanos como políticos estaban acostumbrados — sobre todo en los años setenta y ochenta— a cadáveres semanales, incluso a varios diarios en algunos periodos, hubo ciertos atentados con un especial impacto que pudieron también afectar a la atención política recibida por el terrorismo. El asesinato de Gregorio Ordońez fue uno de ellos. Muy conocido, el entonces líder del PP guipuzcoano, parlamentario vasco y candidato a la alcaldía de San Sebastián, fue el primer político asesinado desde los primeros años ochenta. El atentado tuvo lugar un 23 de enero y el debate sobre el estado de la nación se celebró dos semanas después. Como puede verse en el gráfico 3, parece que su impacto fue considerable, ya que es el dato más alto de toda la serie, aunque también es cierto que es en ese momento cuando la polémica sobre los GAL se encontraba en su punto más álgido, como demuestra el que González dedicara en dicha sesión parlamentaria bastante tiempo a negar las acusaciones que por entonces pesaban contra su Gobierno.

En junio de 1987 ETA cometió su atentado más cruento con bomba en un centro comercial de Barcelona, provocando veintiún muertos. Pocos meses después, en diciembre de ese mismo año, ETA colocó un coche-bomba en la casa cuartel de la Guardia Civil de Zaragoza, asesinando a once personas, entre ellos cinco niñas. Fueron dos atentados que causaron un gran impacto en una opinión pública acostumbrada a ese tipo de noticias por sus especiales características: una masacre de civiles en una gran superficie comercial y la muerte de cinco menores en uno de los atentados más sangrientos cometidos hasta la fecha. En el gráfico podemos ver cómo, efectivamente, el debate de 1988, celebrado dos meses después del atentado de Zaragoza y ocho después del de Hipercor, es el que cuenta con más presencia de asuntos relacionados con el terrorismo hasta esa fecha con casi un $15 \%$ del tiempo. Un muerto más que en Zaragoza hubo en el 
atentado de la Plaza de la República Dominicana, donde ETA asesinó a doce guardias civiles en julio de 1986. Pocos días después tuvo lugar la sesión de investidura del presidente González, en cuyo discurso inicial habló el doble de terrorismo que en su intervención en el debate sobre del estado de la nación del año anterior. Tampoco hay muchas dudas del impacto que causó en la sociedad española la cadena de atentados en cuatro trenes de la red de cercanías de Madrid el 11 de marzo de 2004. En su sesión de investidura celebrada un mes después, José Luis Rodríguez Zapatero dedica al terrorismo un 12,6\% de su discurso, su punto máximo, solo superado en 2007.

En conclusión, sí que parece que atentados especialmente impactantes por diversas características han podido tener un impacto en el tiempo dedicado a hablar del terrorismo por parte de los presidentes de Gobierno españoles. Este resultado es congruente con el obtenido por Criado (2017) para la opinión pública. Según su investigación, el tipo de víctimas que causan los terroristas tiene influencia en la percepción de los espanooles sobre la amenaza terrorista. Así, su preocupación crece si los asesinados son políticos y civiles, más que si son policías o militares. Es decir, que para la opinión pública la tipología de las víctimas importa para la relevancia que otorga al terrorismo, el mismo resultado que hemos obtenido con nuestro análisis preliminar. Obviamente, esta es una primera aproximación cualitativa que tendrá que ser corroborada posteriormente con estudios más exhaustivos que abarquen más fuerzas políticas y que sean capaces de medir de manera objetiva el impacto de los diferentes atentados.

\section{Hipótesis 3. Consenso}

Según los resultados obtenidos por Criado (id.), los acuerdos formales para excluir el terrorismo, en su caso el Pacto de Ajuria-Enea, de la competición política tienen el efecto de reducir la relevancia del terrorismo. Aunque su investigación se refiere a la opinión pública — estudia el porqué de las subidas y bajadas en la preocupación ciudadana por el terrorismo- es lógico pensar que pueda también tener un efecto en la importancia que las élites dan a este tema. Nuestros datos también apoyan esta versión. Así, en los discursos que se pronunciaron con el acuerdo de Ajuria Enea vigente dedicaron de media menos tiempo al terrorismo $(5,6 \%)$ que aquellos que lo fueron con el pacto roto o antes de su firma $(7,6 \%)^{15}$.

\section{Hipótesis 4. Opinión pública}

Cuando los políticos dedican tiempo a una determinada cuestión en sus discursos, ¿están reflejando la importancia que la opinión pública otorga a dicho tema? Para

15. De este cálculo he excluido los discursos de Mariano Rajoy, al no estar ETA ya presente como amenaza en su presidencia. 
comprobarlo he correlacionado el dato de cada discurso con el porcentaje de españoles que en el Barómetro del CIS de dos meses previos a que se pronuncie dicho discurso señalan al terrorismo como uno de los tres principales problemas de España ${ }^{16}$. De esta manera, si existe una relación podremos concluir que una porción del mayor o menor tiempo dedicado al terrorismo por parte de los presidentes de Gobierno viene dictado por el clima de opinión presente en ese momento. La correlación efectivamente es positiva, aunque moderada $(\mathrm{R}=0,562$, sig. $=0,002, \mathrm{~N}=27)$, lo que nos indica que la opinión pública o, al menos, el clima de opinión general, pueden tener influencia en dar o no importancia a la cuestión del terrorismo en los discursos presidenciales. Es decir, lo que el análisis estadístico nos indica es que a mayor preocupación de la opinión pública sobre el terrorismo, mayor tiempo dedicará el presidente de Gobierno en los debates sobre el estado de la nación (y el candidato a presidente en su sesión de investidura) a esta cuestión.

Esta relación, sin embargo, hay que tomarla con mucha cautela. En primer lugar por el escaso número de casos con el que contamos, solo veintisiete. En segundo lugar porque la relación podría ser inversa, es decir, que sea el discurso de los políticos el que influya en la opinión pública: cuanta más importancia dan estos al terrorismo más crece la preocupación en el conjunto de la sociedad. Es cierto que siempre hemos tomado el dato de una encuesta anterior, pero ambos, encuesta y discurso, se encuentran en un contexto mayor en donde el discurso concreto es reflejo de los posicionamientos políticos en ese momento, los cuales pueden haber influido en el dato de la opinión pública que nosotros hemos recogido. Por otro lado, tampoco es descartable una retroalimentación entre ambas variables. En definitiva, los datos con los que contamos en este momento no pueden aclarar dicha relación y es esta una hipótesis que tendrá que ser confirmada en un futuro con más datos y, por supuesto, con un análisis estadístico más sofisticado.

\section{CONCLUSIONES}

El objetivo de esta nota de investigación ha sido dar una respuesta preliminar a la pregunta de cuánto peso tiene y ha tenido el terrorismo en el discurso político de los presidentes de Gobierno en España. Es un dato sin duda relevante, ya que lo podemos considerar un proxy de cuánto tiempo le han dedicado los políticos y, por tanto, de cuánto es el tiempo que no se ha dedicado a otras cuestiones. Se ha realizado mediante

16. Al igual que en el gráfico 1, utilizamos la suma de la multirrespuesta. Por desgracia, en los años setenta, ochenta y noventa no se hacían encuestas con tanta asiduidad como ahora y muchos de los datos no están disponibles en la página web del CIS. Debido a ello para algunos discursos no tenemos su dato correspondiente de opinión pública (hasta 1984; González 1988, y 1989.21993; y Aznar 2000) y en otros no hemos podido escoger justo el dato de dos meses antes, sino el anterior más cercano: mayo de 1985 para el debate de octubre de ese año; octubre de 1985 para la investidura de julio de 1986; junio de 1988 para el debate de febrero de 1989; septiembre de 1993 para el debate de abril de 1994; octubre de 1997 para el debate de mayo de 1998, y marzo de 1999 para el debate de junio de ese año. Los datos se presentan en la tabla A1 del anexo. 
un análisis de contenido manual codificado por el propio autor. Hablamos de una primera aproximación porque, recordamos, en estos momentos solo contamos con datos de los discursos del presidente de Gobierno, a los cuales hemos considerado como una muestra del discurso político en España. Sin embargo, es posible que las conclusiones obtenidas del análisis de los discursos presidenciales no sean aplicables a otros líderes políticos y que en el futuro las conclusiones aquí obtenidas cambien al analizar otro tipo de textos. En cualquier caso, el objetivo de este trabajo es abrir una nueva línea de investigación que tendrá que ser completada a posterior con análisis más amplios, una mayor diversidad de datos y nuevas hipótesis.

Respondiendo a la pregunta antes formulada, se han apuntado algunas hipótesis y posibles líneas de investigación en lo relativo al análisis cuantitativo de textos aplicado al discurso sobre terrorismo. Así, de entrada se ha constatado que una mayor intensidad de los ataques terroristas no hace subir la atención que la élite política pone en dicho tema. Lo que sí hemos visto que puede influir es la impresión que provocan atentados especialmente impactantes. Así, hemos observado cómo los presidentes de Gobierno dedicaban más tiempo de sus discursos tras el asesinato de Gregorio Ordoñez o los atentados del 11-M.

Por otro lado, también hemos señalado cómo las treguas de grupos terroristas, en principio si van acompañadas de "procesos de paz» o de diálogos entre el Gobierno y dichos grupos, hacen crecer la atención dedicada al terrorismo, mientras que la reduce el hecho de que haya un acuerdo formal para excluir a los terroristas de la vida política, en nuestro caso el Pacto de Ajuria-Enea. Finalmente, cuanto más preocupada esté la opinión pública, más atención prestarán los políticos a la violencia política.

\section{Referencias}

Alonso, Rogelio. 2003. Matar por Irlanda. Madrid: Alianza.

Alonso, Rogelio. 2016. "Terrorist skin, peace-party mask: The political communication strategy of Sinn Féin and the PIRA", Terrorism and Political Violence, 28 (3): 520-540. Disponible en: https://doi.org/10.1080/09546553.2016.1155934.

Berrebi, Claude y Esteban F. Klor. 2008. "Are voters sensitive to terrorism? Direct evidence from the Israeli electorate", American Political Science Review, 102 (3): 279301. Disponible en: https://doi.org/10.1017/S0003055408080246.

Casquete, Jesús. 2009. En el nombre de Euskal Herria. Madrid: Tecnos.

Criado, Henar. 2017. "What makes terrorism salient? Terrorist strategies, political competition and public opinion", Terrorism and Political Violence, 29 (2): 197214. Disponible en: https://doi.org/10.1080/09546553.2015.1008628.

De la Calle, Luis e Ignacio Sánchez-Cuenca. 2011. "What We Talk about When We Talk about Terrorism?”, Politics \& Society, 39 (3): 451-472. Disponible en: https:// doi.org/10.1177/0032329211415506.

Domínguez, Florencio. 1998. ETA, estrategia organizativa y actuaciones, 1978-1992. Bilbao: Universidad del País Vasco. 
Domínguez, Florencio. 2003. Las raices del miedo. Madrid: Aguilar.

Domínguez, Florencio. 2012. La agonía de ETA: una investigación inédita sobre los últimos días de la banda. Madrid: La Esfera de los Libros.

Domínguez, Florencio y Gaizka Fernández (eds.). 2018. Pardines. Cuando ETA empezó a matar. Madrid: Tecnos.

Elorza, Antonio (coord.). 2000. La historia de ETA. Madrid: Temas de Hoy.

Fernández Soldevilla, Gaizka. 2013. Héroes, heterodoxos y traidores: historia de Euskadiko Ezkerra (1974-1994). Madrid: Tecnos.

Fernández Soldevilla, Gaizka. 2016. La voluntad del "gudari": génesis y metástasis de la violencia de ETA. Madrid: Tecnos.

Fernández Soldevilla, Gaizka y Raúl López Romo. 2012. Sangre, votos, manifestaciones: ETA y el nacionalismo vasco radical (1958-2011). Madrid: Tecnos.

Garmendia, José María. 1980. Historia de ETA. San Sebastián: Haramburu.

Geifman, Anna. 2013. "The liberal opts for terror", Terrorism and Political Violence, 25 (4): 550-560. Disponible en: https://doi.org/10.1080/09546553.2013.814494.

Grimmer Justin y Brandon M. Stewart. 2013. "Text as data: The promise and pitfalls of automatic content analysis methods for political texts", Political Analysis, 21 (3): 267-297. Disponible en: https://doi.org/10.1093/pan/mps028.

Herschinger, Eva. 2013. "A battlefield of meanings: The struggle for identity in the UN debates on a definition of international terrorism", Terrorism and Political Violence, 25 (2): 183-201. Disponible en: https://doi.org/10.1080/09546553.2011.652318.

Hetherington, Marc J. y Elisabeh Suhay. 2011. "Authoritarianism, threat and Americans's support for the war on terror", American Journal of Political Science, 102 (3): 546-560. Disponible en: https://doi.org/10.1111/j.1540-5907.2011.00514.x.

Jáuregui, Gurutz. 1981. Ideología y estrategia politica de ETA. Madrid: Siglo XXI.

Kibris, Arzu. 2011. "Funerals and elections: The effects of terrorism on voting behavior in Turkey", Journal of Conflict Resolution 55 (2): 220-247. Disponible en: https://doi.org/10.1177/0022002710383664.

Laver, Michael (ed.). 2001. Estimating the policy position of political actors. Londres: Routledge.

Leonisio, Rafael. 2015. "Ida y vuelta. El discurso de Euskal Herritarrok sobre la violencia en la tregua de Lizarra”, Inguruak, 59: 1-22.

Leonisio, Rafael, Fernando Molina y Diego Muro (eds.). 2017. ETA's terrorist campaign: from violence to politics, 1968-2015. Londres: Routledge.

Llera, Francisco. 1992. "Violencia y opinión pública en el País Vasco: 1978-1992", Revista Internacional de Sociología, 3: 83-111.

Llera, Francisco. 2012. "Terrorismo y opinión pública en España”, en Ubaldo Cuesta, María José Canel y Mario Gurrionero (eds.), Comunicación y terrorismo. Madrid: Tecnos.

Llera, Francisco. 2013. "ETA: medio siglo de terrorismo y limpieza étnica en Euskadi”, Sistema, 231: 3-46.

Llera, Francisco y Rafael Leonisio. 2017. "La estrategia del miedo. ETA y la espiral del silencio en el País Vasco", Informe del Centro Memorial de Victimas del Terrorismo, 1. 
López Romo, Raúl. 2015. Informe Foronda. Los efectos del terrorismo en la sociedad vasca. Madrid: Libros de la Catarata.

Martin-Peña, Javier y Susan Opotow. 2011. "The Legitimization of Political Violence: A Case Study of ETA in the Basque Country, Peace and Conflict", Journal of Peace Psychology, 17 (2): 132-150. Disponible en: https://doi.org/10.1080/107 81919.2010.550225.

Mcevoy, Kieran, Peter Shirlow y Karen Mcelrath. 2004. "Resistance, transition and exclusion: politically motivated ex-prisioners and conflict transformation in Northern Ireland", Terrorism and Political Violence, 16 (3): 646-670. Disponible en: https://doi.org/10.1080/09546550490509991.

McGovern, Mark. 2004. "The old days are over. Irish republicanism, the peace process and the discourse equality", Terrorism and Political Violence, 16 (3): 622-645. Disponible en: https://doi.org/10.1080/09546550490509955.

Mees, Ludger. 2003. Nationalism, Violence and Democracy. The Basque Clash of Identities. Nueva York: Palgrave Macmillan. Disponible en: https:/doi. org/10.1057/9781403943897.

Muro, Diego. 2008. Ethnicity and Violence. The Case of Radical Basque Nationalism. Nueva York: Routledge.

Reinares, Fernando. 2001. Patriotas de la muerte. Madrid: Taurus.

Sánchez-Cuenca, Ignacio. 2010. "La pervivencia del terrorismo de ETA", en Antonio Rivera y Carlos Carnicero Herreros (eds.), Violencia Politica: historia, memoria y victimas. Madrid: Maia.

Schmid, Alex P. 2010. "Frameworks for conceptualising terrorism", Terrorism and Political Violence, 16 (2): 197-221. Disponible en: https://doi.org/10.1080/ 09546550490483134.

Sullivan, John. 1988. ETA and Basque Nationalism. The Fight for Euskadi, 1890-1986. Nueva York: Routledge.

Thorup, Mikkel. 2009. "Enemy of humanity: The anti-piracy discourse in present-day anti-terrorism", Terrorism and Political Violence, 21 (3): 401-411. Disponible en: https://doi.org/10.1080/09546550902950282.

Volkens, Andrea. 2007. "Strengths and weaknesses of approaches to measuring policy positions of parties”, Electoral Studies 26 (1): 108-120. Disponible en: https://doi. org/10.1016/j.electstud.2006.04.003.

Watson, Cameron J. 2008. Basque Nationalism and Political Violence: The Ideological and Intellectual Origins of ETA. Reno: University of Nevada Press.

Weinberg, Leonard, Ami Pedahzur y Sivan Hirsch-Hoefler. 2011. "The Challenges of Conceptualizing Terrorism", Terrorism and Political Violence, 16 (4): 777-794. Disponible en: https://doi.org/10.1080/095465590899768.

Whitfield, Teresa. 2014. Endgame for ETA. Elusive Peace in the Basque Country. Nueva York: Hurst \& Co.

Whiting, Sophie A. 2012. "The discourse of defence: Dissident Irish Republican newspapers and the propaganda war", Terrorism and Political Violence, 24 (3): 483-503. Disponible en: https://doi.org/10.1080/09546553.2011.637587. 


\section{ANEXOS}

Tabla Ai.

PorCentaje de TEXTO DEDICADO AL TERRORISMO POR CADA PRESIDENTE DE GOBIERNO (DEBATES SOBRE EL ESTADO DE LA NACIÓN) O CANDIDATOS A SERLO (SESIONES DE INVESTIDURA)

\begin{tabular}{|c|c|}
\hline 1979 Suárez & 2,55 \\
\hline 1981 Calvo Sotelo & 6,29 \\
\hline 1982 González & 3,17 \\
\hline 1983 González & 5,63 \\
\hline 1984 González & 4,48 \\
\hline 1985 Gonzales & 4,72 \\
\hline 1986 González & 8,81 \\
\hline 1987 González & 10,12 \\
\hline 1988 González & 13,09 \\
\hline 1989. 1 González & 3,47 \\
\hline 1989.2 González & 7,22 \\
\hline 1991 González & 2,35 \\
\hline 1992 González & 4,93 \\
\hline 1993 González & 0,74 \\
\hline 1994 González & 0,42 \\
\hline 1995 González & 17,07 \\
\hline 1996 Aznar & 2,95 \\
\hline 1997 Aznar & 3,53 \\
\hline 1998 Aznar & 5,95 \\
\hline 1999 Aznar & 12,53 \\
\hline 2000 Aznar & 7,05 \\
\hline 2001 Aznar & 9,57 \\
\hline 2002 Aznar & 15,07 \\
\hline 2003 Aznar & 16,51 \\
\hline 2004 Zapatero & 12,63 \\
\hline 2005 Zapatero & 9,55 \\
\hline 2006 Zapatero & 2,4 \\
\hline 2007 Zapatero & 13,03 \\
\hline 2008 Zapatero & 4,6 \\
\hline 2009 Zapatero & 2,06 \\
\hline
\end{tabular}




\begin{tabular}{ll}
$\ldots / \ldots$ & \\
\hline 2010 Zapatero & 3,74 \\
\hline 2011 Zapatero & 4,68 \\
\hline 2011.1 Rajoy & 0,2 \\
\hline 2013 Rajoy Rajoy & 0 \\
\hline 2015 Rajoy & 0 \\
\hline 2016. 1. Sanchez & 2,77 \\
\hline 2016.2. Rajoy & 1,7 \\
\hline
\end{tabular}

Fuente: elaboración propia.

En la tabla A2 se pueden encontrar las cincuenta palabras más frecuentes de las partes del texto que se ha codificado manualmente como terrorismo. Como puede verse, la mayoría de palabras con contenido político explícito (terrorismo, violencia, terroristas, ETA, paz, lucha...) están relacionadas con la cuestión del terrorismo. De esta manera podemos confirmar que la codificación manual se ha realizado de manera adecuada y que por tanto los porcentajes obtenidos son válidos.

TABLA A2.

Palabras más FReCUENTES EN los teXtos anAlizados

\begin{tabular}{lclclc}
\hline \multicolumn{1}{c}{ Palabra } & Frecuencia & \multicolumn{1}{c}{ Palabra } & Frecuencia & \multicolumn{1}{c}{ Palabra } & Frecuencia \\
\hline Terrorismo & 215 & Acción & 42 & País & 27 \\
\hline Gobierno & 123 & Libertad & 41 & Puede & 27 \\
\hline Contra & 116 & Víctimas & 41 & Derechos & 25 \\
\hline Todos & 100 & Ciudadanos & 38 & Libertades & 25 \\
\hline Lucha & 83 & Cámara & 38 & Constitución & 24 \\
\hline Violencia & 76 & ETA & 37 & Eficacia & 24 \\
\hline Estado & 74 & Españoles & 34 & Ley & 24 \\
\hline Seguridad & 65 & España & 33 & Tiene & 24 \\
\hline Fuerzas & 62 & Paz & 33 & Toda & 24 \\
\hline Internacional & 59 & Apoyo & 32 & Democracia & 23 \\
\hline Política & 56 & Derecho & 32 & Político & 23 \\
\hline Terrorista & 52 & Ańos & 29 & Vasco & 23 \\
\hline Señorías & 50 & Democrático & 29 & Antiterrorista & 22 \\
\hline Cooperación & 49 & Hoy & 29 & Voluntad & 22 \\
\hline Terroristas & 47 & Políticas & 29 & Fenómeno & 21 \\
\hline Quiero & 44 & Compromiso & 28 & Fin & 21 \\
\hline Sociedad & 43 & Acuerdo & 29 & & \\
\hline
\end{tabular}

Nota: se muestran las cincuenta primeras palabras. Se han excluido las preposiciones, determinantes, pronombres, conjunciones, verbos auxiliares etc.

Fuente: elaboración propia. 
Finalmente, hemos validado nuestra codificación manual mediante la comparación de nuestros resultados con los obtenidos de aplicar un diccionario de palabras (otra de las técnicas del análisis de contenido) a los mismos textos que hemos codificado de manera manual. Como puede verse en el gráfico A1, la correlación es casi perfecta $(R=0,93, N=38)$ entre los resultados de ambos tipos de técnicas; lo que nos indica que nuestra codificación manual se ha realizado de manera correcta y que los resultados que hemos obtenido reflejan efectivamente el porcentaje de tiempo de dedicado al terrorismo en cada discurso.

Gráfico Ai.

COMPARACIÓN ENTRE LA CODIFICACIÓN MANUAL Y LA APLICACIÓN DE UN DICCIONARIO

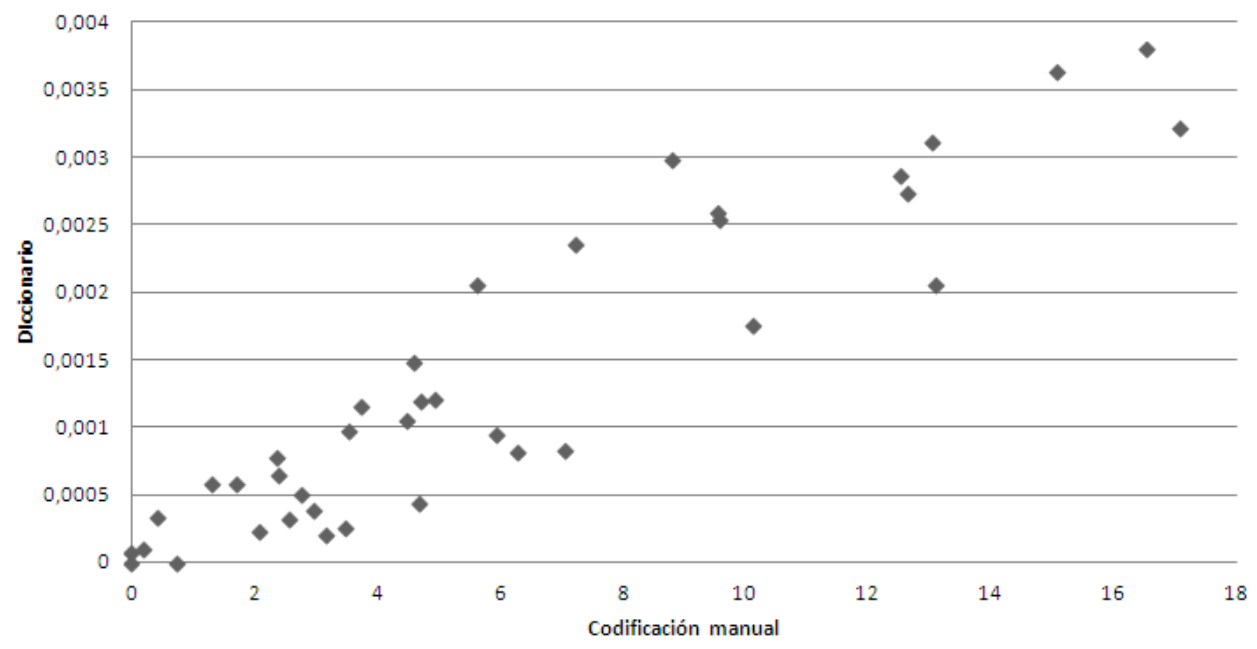

Fuente: elaboración propia.

Palabras relativas al terrorismo empleadas para la elaboración del diccionario:

"ETA"; "GAL”;“ajuria”;“Ajuriaenea”;"antiterro*”;"asesin*”;"atentado*”;"bomba*”;"borroka";"callejer*”;"comando”;"contramanifes*”;"dispersión”;"enea”;"escol-

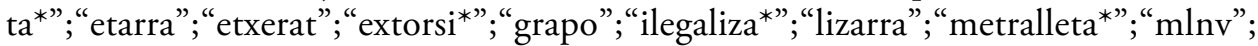
"paz";"santuario";"secuestr*”;"terror*”;“tortura*”;"tregua"; yihad*”

Presentado para evaluación: 15 de junio de 2018. Aceptado para publicación: 4 de febrero de 2019.

\section{RAFAEL LEONISIO}

rafael.leonisio@ehu.eus

Investigador postdoctoral en el departamento de Ciencia Política de la UPV-EHU y colaborador con el equipo de investigación del Regional Manifestos Project de la 
Universidad de Deusto. Es autor de Cambio y continuidad en el discurso político: el caso del partido socialista de Euskadi (1977-2011) (CIS, 2016) y coeditor de ETA's terrorist campaign: from violence to politics, 1968-2015 (Routledge, 2017). Sus áreas de interés se centran en los partidos políticos, el comportamiento electoral, la violencia política y el análisis de contenido, cuestiones sobre las que ha publicado en revistas como European Journal of Political Research, National Identities, Regional \& Federal Studies, REIS, Revista Española de Ciencia Politica o Revista de Estudios Politicos. Es miembro del consejo de redacción de la revista Inguruak. 